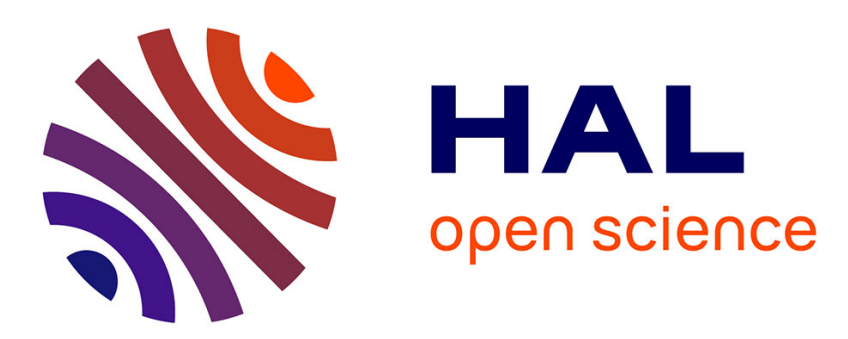

\title{
Photodegradation of azobenzene nonlinear optical chromophores: the influence of structure and environment
}

\author{
A. Galvan-Gonzalez, Michael Canva, G.I. Stegeman, Kwok Pong Chan, Tony \\ C. Kowalczyk, Hilary S. Lackritz
}

\section{To cite this version:}

A. Galvan-Gonzalez, Michael Canva, G.I. Stegeman, Kwok Pong Chan, Tony C. Kowalczyk, et al.. Photodegradation of azobenzene nonlinear optical chromophores: the influence of structure and environment. Journal of the Optical Society of America B, 2000, 17 (12), pp.1992-2000. hal-00668000

\section{HAL Id: hal-00668000 \\ https://hal-iogs.archives-ouvertes.fr/hal-00668000}

Submitted on 8 Feb 2012

HAL is a multi-disciplinary open access archive for the deposit and dissemination of scientific research documents, whether they are published or not. The documents may come from teaching and research institutions in France or abroad, or from public or private research centers.
L'archive ouverte pluridisciplinaire HAL, est destinée au dépôt et à la diffusion de documents scientifiques de niveau recherche, publiés ou non, émanant des établissements d'enseignement et de recherche français ou étrangers, des laboratoires publics ou privés. 


\title{
Photodegradation of azobenzene nonlinear optical chromophores: the influence of structure and environment
}

\author{
Adriana Galvan-Gonzalez, Michael Canva,* and George I. Stegeman \\ School of Optics/Center for Research and Education in Optics and Lasers, University of Central Florida, \\ P.O. Box 162 700, 4000 Central Florida Boulevard, Orlando, Florida 32816-2700 \\ Ludmila Sukhomlinova and Robert J. Twieg \\ Department of Chemistry, Kent State University, Kent, Ohio 44242
}

Kwok Pong Chan

Molecular OptoElectronics Corporation, 877 25th Street, Watervliet, New York 12189-1903

Tony C. Kowalczyk and Hilary S. Lackritz ${ }^{\dagger}$

Gemfire Corporation, Suite 600, 2471 East Bayshore Road, Palo Alto, California 94303

Received April 17, 2000; revised manuscript received July 18, 2000

\begin{abstract}
The photodegradation of a variety of donor-acceptor-substituted azobenzene nonlinear optical chromophores has been studied. The variables examined that influence the rate of degradation include chromophore and host polymer structure, irradiation wavelength, temperature, and atmosphere. (C) 2000 Optical Society of America [S0740-3224(00)01311-4]

OCIS codes: $160.4330,160.2100,190.4400,260.5130$.
\end{abstract}

\section{INTRODUCTION}

Azobenzene dye molecules that are functionalized with a pair of electron-donor and electron-acceptor groups have attractive properties for a variety of optical and nonlinear optical applications. ${ }^{1-6}$ The conjugated azobenzene structure facilitates electron transfer between the donor and acceptor groups, resulting in a charge-transfer excited state. The optically induced transition between the ground state and this excited state leads to an intense absorption feature in the blue-green region of the visible spectrum. Not only is the transition dipole moment large but these molecules also possess both a large groundstate dipole moment and a large first hyperpolarizability. These molecular characteristics lead to the creation of large macroscopic second-order nonlinear coefficients when the molecules are aligned in a polar fashion. When such chromophores are incorporated into polymers, the polar order is created by electric field poling near the polymer's glass-transition temperature at which the polymer becomes rubbery and facilitates partial rotational alignment of the chromophores by means of the molecular dipole-external field interaction. The resultant optical nonlinearity has proved attractive for electro-optic and frequency conversion applications.

One of the real barriers to adoption of such polymers for optical applications is the fact that under illumination a fraction of the chromophores excited into the chargetransfer state does not return to the original ground-state structure. One possible outcome is the isomerization of the thermodynamically more stable trans geometry of the azobenzene to the cis geometry. This reversible photoisomerization is, in fact, the basis for a variety of novel and useful applications: photopoling, optical storage, grating formation, and many others. ${ }^{2,3,5,6}$ However, other, nonreversible, processes that involve destruction of the chromophore and associated optical damage of the surrounding guiding medium may also result. ${ }^{7-9}$ The new altered chemical structure likely involves some reduction of the electron transfer properties of the conjugated molecule or changes in the characteristics of the donor or acceptor groups that are responsible for the optical nonlinearity. ${ }^{10,11}$ Although some useful applications (such as bleaching to create a waveguide) that involve permanent photodegradation might be imagined, almost invariably this change represents a serious problem for most photonic applications. ${ }^{12,13}$

In this paper we investigate the photodegradation of a number of electro-optic materials that contain an azobenzene guest chromophore in a host polymer. More specifically, we examine eight different azo dyes with a variety of donor-acceptor combinations including some of the most important ones for electro-optic applications (amine donors with cyano or nitro acceptors). Investigations in different gaseous environments are selectively used to elucidate the role of different degradation mechanisms. We also investigate how the host polymer influences photodegradation by examining two different azobenzenes in three common host matrices, namely, poly(methyl 
methacrylate) (PMMA), a polyethersulfone (PES), and a polyimide (Ultem). In each case, a figure of merit related to the decay rate for a particular chromophore was measured at a number of wavelengths from 442 to $1320 \mathrm{~nm}$. The systems studied here certainly do not comprehensively represent the large array of structures already studied and currently under development but do represent some of the generic challenges associated with photochemical stability of electro-optic materials.

\section{CHROMOPHORES AND POLYMERS INVESTIGATED}

Although side-chain polymers are most frequently used for electro-optic devices, previous investigations of the stilbene 4- $N, N$-dimethylamino-4'-nitrostilbene (DANS) and azobenzene Disperse Red 1 (DR1) chromophores in both guest-host and side-chain polymer formats showed similar behavior, with the side chain being more stable by as much as 1 order of magnitude. ${ }^{10,11,14,15}$ Inasmuch as a guest-host system does not require chemical attachment of the chromophore to a host polymer, it can be fabricated more readily than side chain systems. One generally prepares guest-host systems by simply mixing a nonlinear-optic chromophore that possesses a large molecular hyperpolarizability into an amorphous polymer matrix. Thus one can prepare a variety of systems by mixing two components together, although the chromophore concentrations are typically low because of solubility and aggregation effects that are known to occur., ${ }^{9,16}$ The nonlinear-optical dyes studied here were dissolved into the polymer host with a common solvent (propyleneglycolmethyletheracetate), and thin films were dried in vacuum for evaporation of the remaining solvent. Our guest-host systems typically contained $\sim 5 \%$ by weight of the chromophore. First, each fused-silica substrate was coated with a thin aluminum film into which round holes $50-250 \mu \mathrm{m}$ in diameter were fabricated photolithographically to provide uniform and reproducible illumination onto the polymer film. The polymer samples were spun onto the glass surface opposite that of the metal film. The optical beams were incident through the holes, the glass, and then onto the back side of the polymer film.

The structures of the chromophores and the host polymers, the location of the absorption maximum owing to excitation of charge-transfer state $\lambda_{\max }$, and the oxidation potentials for the chromophores are listed in Table 1 . Many of the chromophores are known substances and were synthesized by the coupling of a diazonium salt with an aniline derivative, except for chromophore 3, which was prepared directly from DR1 (chromophore 1), which is itself commercially available. All new compounds were prepared in an analogous fashion. The oxidation potentials were determined with a platinum electrode and at a millimolar concentration containing a $0.1 \mathrm{M}$ solution of tetraethylammonium tetrafluoroborate versus standard calomel electrode/saturated sodium choloride in a three-electrode cell at a scan rate of $300 \mathrm{mV} / \mathrm{s}$. All the electrochemical oxidations are at least partially reversible, but only the values $E_{p}{ }^{1}$ of the potential at the maximum of the first oxidation wave are provided. Many of
Table 1. Chemical Structures of the Chromophores Studied, with Their Values of $\lambda_{\max }$, $B$ Measured at $633 \mathrm{~nm}$, Parameters $D_{0}$ and $E_{0}$, and Oxidation Potentials ${ }^{a}$

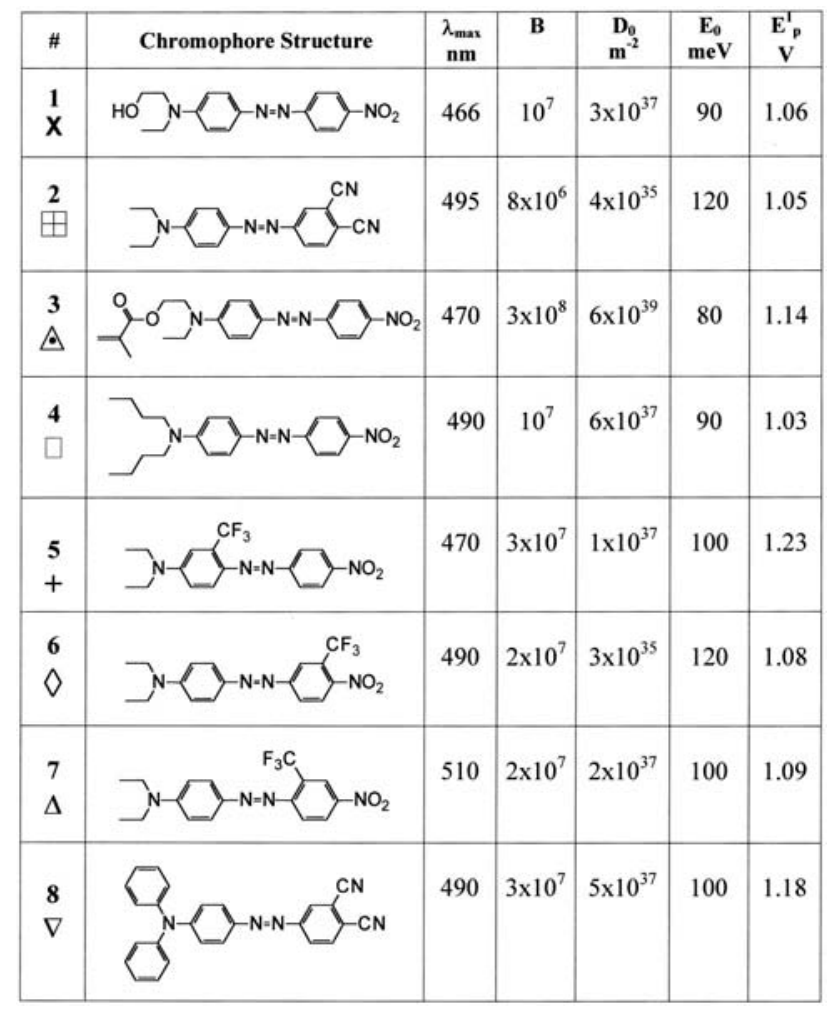

${ }^{a}$ \#, Chromophore number used in text. Symbols beneath numbers identify symbols used in Figs. 5-10 and 13.

the same azodyes had been evaluated earlier in an investigation of the influence of structure on thermal stability. ${ }^{17-20}$

\section{PHOTODEGRADATION MODEL AND EXPERIMENTAL TECHNIQUES}

There are multiple photodegradation channels available for any chromophore or polymer because in principle many chemical bonds in a molecule can be affected by the absorption of light, although just a few may initially be the most sensitive. However, our (and others') experience has shown that there are usually only a few dominant channels, which are practically limited to no more than two when the response of the material in the near infrared is of interest. ${ }^{10,11,14,15}$ So we assume that such is the case at the outset and adopt the model shown in Fig. 1. ${ }^{21}$ Light of wavelength $\lambda$ and photon energy $E_{\text {phot }}$ $=h \nu$ is absorbed (molecular absorptivity), and the molecule is raised to the charge-transfer excited state. A small fraction, $B^{-1}$, of the molecules undergoes a transformation and ends up in the ground state of a different molecule or geometrical conformation from which no backconversion to the original species is possible. Writing the concentration of the original azobenzene molecules as $N_{1}$ and the sum of the new species as $N_{2}$, we find the rate of change in the concentration of the new species, 


$$
\frac{\partial N_{2}(t)}{\partial t}=-\frac{\partial N_{1}(t)}{\partial t}=\left[\frac{B}{\sigma}\right] N_{1}(t) n(t) \propto-\frac{\partial \alpha_{1}(t)}{\partial t},
$$

where $n(t)$ is the number of photons per $\mathrm{s}$ that pass through a unit area of the sample, $\sigma$ is the molecular absorptivity, and $\alpha_{1}$ is the macroscopic absorption coefficient of the polymer, where it has been explicitly assumed that there is no spectral structure of the host polymer that overlaps either the charge-transfer absorption peak or wavelengths longer than $\lambda_{\max }$. Clearly the parameter $[B / \sigma] n(t)$ plays the role of a lifetime for the active species illuminated with a photon flux $n(t)$. Furthermore, $B / \sigma$ is effectively the lifetime of the electro-optic activity of a molecule per unit incident photon flux, and we define this quantity as the molecular figure of merit (FOM) that is characteristic of the photodegradation rate of the polymer and is dependent on both the chromophore and its environment. The larger the ratio $B / \sigma$, the more photochemically stable the polymer is.

On physical grounds one would expect the quantum efficiency $B^{-1}$ to be independent of photon energy for a single dominant charge-transfer state with a single decay channel, especially in the low-energy tail of the absorption line. We write the absorptivity as $\sigma=\sigma_{0} f\left(E_{\text {phot }}\right.$ - $h c / \lambda_{\max }$ ), where $\sigma_{0}$ is the peak molecular absorptivity associated with the charge-transfer state located at $\lambda_{\max }$; $f\left(E_{\text {phot }}-h c / \lambda_{\max }\right)$ describes the spectral distribution of the absorption, i.e., the line shape of the absorption, and $E_{\text {phot }}$ is the photon energy:

$$
B / \sigma=B /\left[\sigma_{0} f\left(E_{\text {phot }}-h c / \lambda_{\max }\right)\right] .
$$

Therefore one would expect the photon energy dependence of $B / \sigma$ to be influenced by the spectral shape of the absorption spectrum. It has been shown that the nearinfrared tail of the absorption spectrum of many guesthost polymers varies as $\exp \left[-\left(E_{\text {phot }}-h c / \lambda_{\max }\right) / E_{0}\right] .^{22,23}$ Here $E_{0}$ is a constant that varies with both the host polymer and the chromophore; the smaller $E_{0}$ is, the spectrally narrower is the tail of the absorption spectrum and the faster the figure of merit increases with decreasing photon energy (i.e., increasing wavelength). That is, $E_{0}$ is a broadening term that influences how far out in the

\section{Molecule 1}

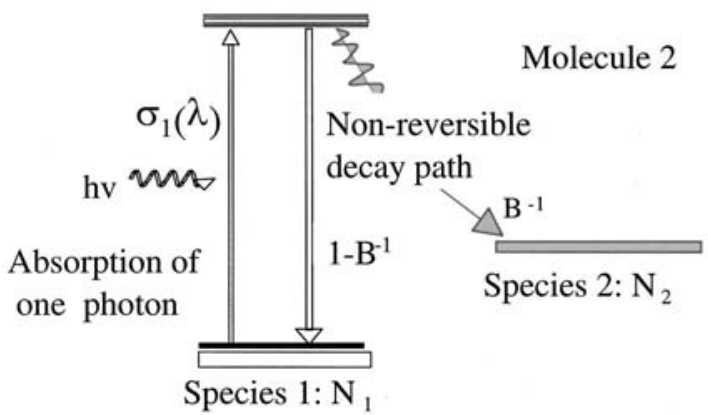

Fig. 1. Model for the photodegradation process that involves raising initial Molecule 1 with concentration $N_{1}$ to an excited state by means of the absorption of a photon (molecular absorptivity $\sigma$ ) followed by decay with quantum efficiency $B^{-1}$ to the new species Molecule 2 (concentration $N_{2}$ ) or by relaxation back to the ground state of Molecule 1 with quantum efficiency $1-B^{-1}$

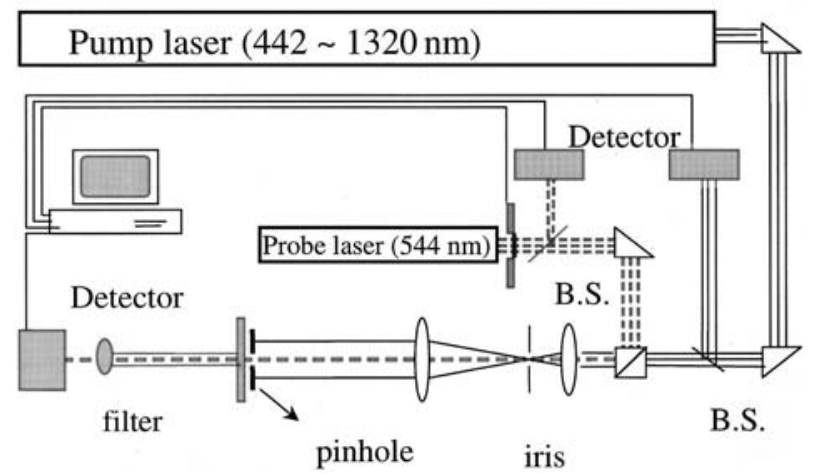

Fig. 2. Schematic of the experimental apparatus employed for the photodegradation studies. B.S.'s, beam splitters.

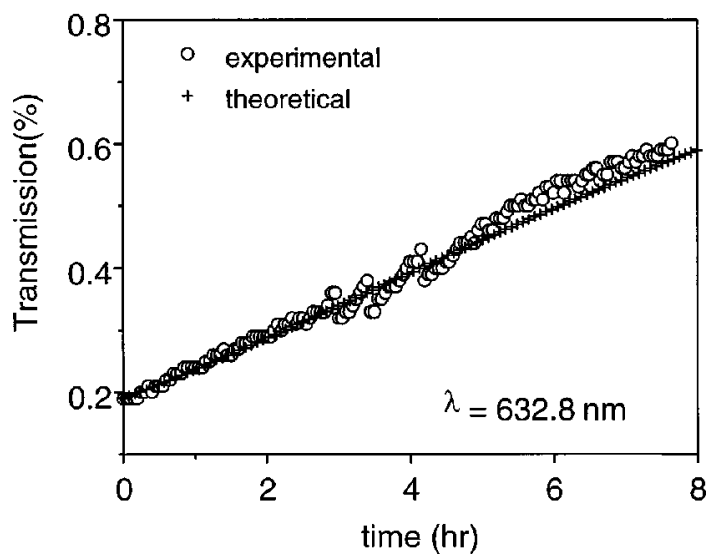

Fig. 3. Change in the transmission of a 3.2- $\mu \mathrm{m}$-thick film of 5 wt. \% of 4- $N, N$-diethylamino- $3^{\prime}, 4^{\prime}$-dicyanoazobenzene (chromophore 2) in a PMMA host polymer with a probe at $544 \mathrm{~nm}$ and a pump at $633 \mathrm{~nm}$ with an intensity of $0.24 \mathrm{~W} / \mathrm{cm}^{2}$.

infrared the degradation is a serious problem. This behavior typically reflects the degree of inhomogeneous broadening of the system; therefore

$$
\begin{aligned}
B / \sigma & =B / \sigma_{0} \exp \left[\left(E_{\text {phot }}-h c / \lambda_{\max }\right) / E_{0}\right] \\
& =D_{0} \exp \left(E_{\text {phot }} / E_{0}\right) .
\end{aligned}
$$

Here $D_{0}$ is the lifetime per unit of photon flux. Under these assumptions, the parameters $D_{0}$ and $E_{0}$ should be independent of wavelength, and it proves convenient to characterize the wavelength dependence of $B / \Phi$ in the near infrared by these two parameters.

The apparatus used in the measurements is shown in Fig. 2. We periodically monitored the change in the active chromophore concentration that resulted from photodegradation by measuring the sample transmission with a $\mathrm{He}-\mathrm{Ne}$ laser at $544 \mathrm{~nm}$. This laser line overlaps the main absorption band of the azobenzene chromophore in its long-wavelength tail. As the number of intact molecules per unit volume decreases, the transmission of the probe beam increases, and this is the quantity measured in our experiments. A typical example is shown in Fig. 3 for compound 2 of Table 1 (4- $N, N$-diethylamino$3^{\prime}, 4^{\prime}$-dicyanoazobenzene). Note the initial straight-line behavior of the transmission with cumulative flux (time), 
as predicted by expression (1). This slope yields a direct measure of $B / \sigma$ and hence of the lifetime for a given photon flux.

A number of different lasers were used as the pump source; these lasers included Nd:YAG for 1320 and 1064 $\mathrm{nm}$, Ti:sapphire for the range $750-900 \mathrm{~nm}, \mathrm{He}-\mathrm{Ne}$ for 633 and $544 \mathrm{~nm}$, an Ar-ion laser for wavelengths from 450 to $530 \mathrm{~nm}$, and a He-Cd for wavelengths down to $450 \mathrm{~nm}$. The pump beam was expanded such that the illumination through the aperture was uniform. Various polarizations were used, and the results were in agreement with the model. ${ }^{21}$ Finally, we verified that the initial slope was linear with respect to increase in the input flux, ruling out any thermal enhancement of the photodegradation efficiency owing to sample heating by absorption or any other nonlinear absorption mechanism.

It is useful to examine whether there is evidence in the azobenzene dyes for more than one excited state's being effectively photobleached. That is, does more than one absorption peak in the visible or the near ultraviolet change with continued exposure to visible and nearinfrared radiation? It is known that, in the nitrostilbene, DANS, illumination in the visible leads to changes in two absorption bands in the visible and the near ultraviolet. ${ }^{15}$ The changes in the absorption spectrum with time that result from irradiation at $497.5 \mathrm{~nm}$ are shown in Fig. 4 for 4-N,N-diethylamino- $3^{\prime}-4^{\prime}$-dicyanoazobenzene (chromophore 2; Table 1). Based on the previous research with azobenzene DR1, two effects, namely, a reversible transcis isomerization and an irreversible photo-oxidation, occur simultaneously. ${ }^{11}$ Note that the absorption peak near $490 \mathrm{~nm}$ associated with the dominant chargetransfer state is strongly photobleached. The second, weaker, peak near $300 \mathrm{~nm}$ is the second, weaker, chargetransfer state that occurs at higher energy in azobenzenes. This peak apparently first grows in strength and then decreases with continued illumination. We speculate that this sequence of events is associated with the cis form created through trans-cis isomerization. The subsequent decrease of this spectral feature may also be due to photo-oxidation. The number of molecules of compound 2 with the strong charge-transfer state is dimin-

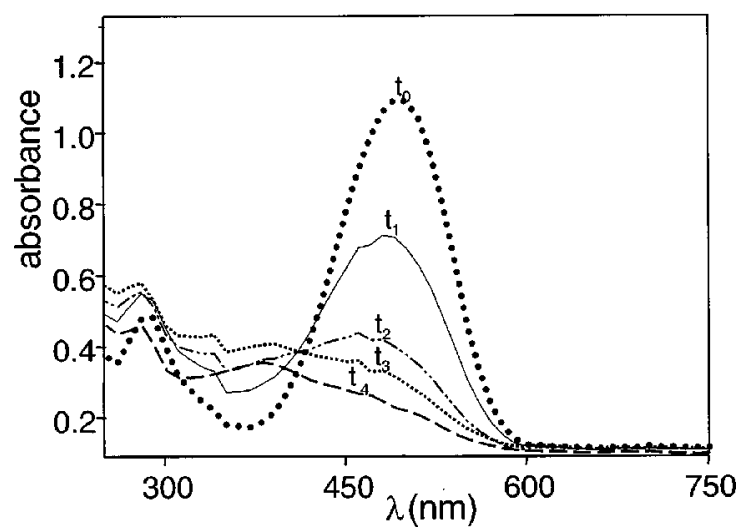

Fig. 4. Evolution of the absorption spectrum of a 3.17- $\mu$ m-thick film of 5 wt. \% 4-N,N-diethylamino- $3^{\prime}, 4^{\prime}$-dicyanoazobenzene (chromophore 2) in a PMMA host polymer on irradiation in air at $497 \mathrm{~nm}$. Here $t_{0}, t_{1}, t_{2}, t_{3}$, and $t_{4}$ correspond to irradiation times of $0,30,60,90$, and $120 \mathrm{~min}$, respectively. ished by both processes, a behavior that is identical to that seen in DR1. ${ }^{11}$ The single-channel decay assumption is not completely adequate in azobenzenes; however, based on the results for DR1, the behavior of the photodegradation for $\lambda>\lambda_{\max }$ is probably dominated by a single decay pathway.

\section{EFFECTS OF DIFFERENT DONORS AND ACCEPTORS}

The different azobenzene-based chromophores that have been incorporated into the host polymer PMMA for this study are listed in Table 1 . The results of some relative stability experiments are shown graphically in Figs. 5-7.

Figure 5 shows the influence of donor modifications of the well-known DR1 structure (chromophore 1) that has a nitro acceptor and an amine donor for which is substituted an ethyl group and a 2-hydroxyethylamino group. The nitro acceptor group is kept constant, and the hydroxyl group in chromophore 1 is esterified with methacrylic acid to yield Disperse Red 1-methacrylate (DR1-M; chromophore 3). Chromophore 3 is, in fact, the reactive monomer that is employed for the preparation of polymethacrylates. The 4-N,N-dibutylamino- 4 '-nitroazobenzene (chromophore 4) again has the nitro acceptor group, but here a pair of simple $n$-butyl groups substitutes for the amine donor nitrogen atom.

The trends and general features of the relationship of $B / \sigma$ and photon energy (or wavelength) are similar for chromophores 1, 2, and 4 and are also similar to those observed in all the other electro-optic polymers that we have investigated to date. ${ }^{10}$ This FOM exhibits plateaulike behavior near the absorption band (3.0-2.0 eV), rises linearly $(2.0-1.5 \mathrm{eV})$ with decreasing photon energy on $\log [B / \sigma]$ versus $E_{\text {phot }}$, and then deviates sharply from this straight line at $0.95 \mathrm{eV}(1320 \mathrm{~nm})$, leading in some cases to a stability value lower at $0.95 \mathrm{eV}$ than at $1.17 \mathrm{eV}(1064$ $\mathrm{nm})$. The key point overall is that the photostability increases by almost 7 orders of magnitude between the visible and $1000 \mathrm{~nm}$. As was pointed out previously, this increase in relative stability is a direct reflection of the decrease in the absorption coefficient with increasing wavelength. ${ }^{10}$ The decrease instability in the region about $1320 \mathrm{~nm}$ has been attributed to generation of singlet oxygen that leads to enhanced photodegradation in DANS. The decreased stability in the azobenzene family at this wavelength can also be attributed the involvement of singlet oxygen. The photochemical generation, the lifetime, and the reactions of singlet oxygen are highly complicated and have been implicated in the degradation of many organic materials, although detailed experimental verification has been available only relatively recently, as in the case of phenylenevinylene systems. ${ }^{24}$

It is rather surprising that, among the eight chromophores studied here, compound 3 is the most photochemically stable. The methacrylate group in compound 3 is the unique (and rather unorthodox) feature in this molecule and must have some role in the molecule's extra photostability, perhaps acting as a so-called getter by preferentially interacting with or scavenging oxygen. The role of oxygen in the polymerization of vinyl compounds, such as the acrylates, is also known to be compli- 


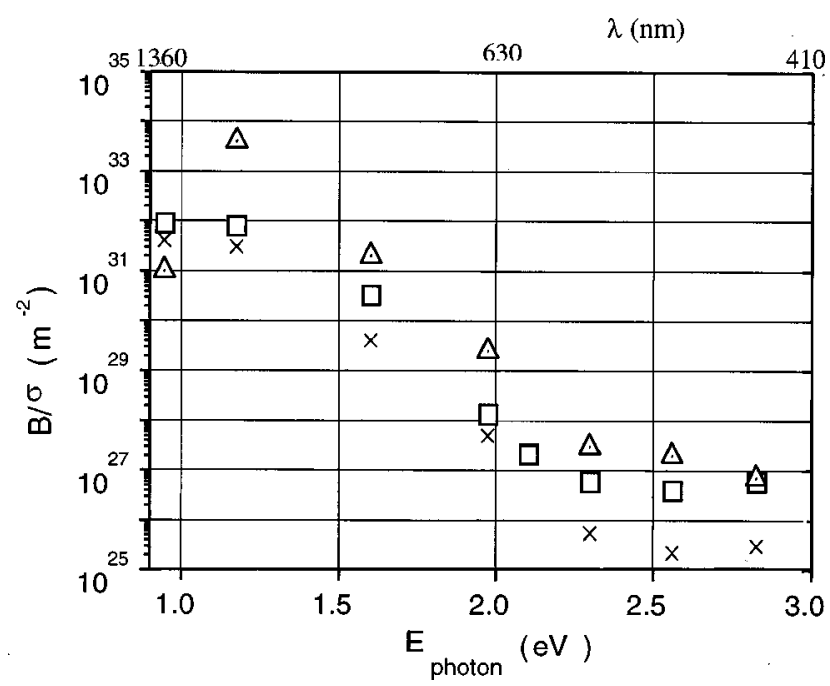

Fig. 5. Dependence of photostability FOM $B / \sigma$ on the photon energy for three different guest chromophores, 4-(N-ethyl- $N$-2hydroxyethyl)-4' -; nitroazobenzene (DR1 chromophore $1 ; \times$ ), DR1-M; chromophore 3 ; ( $\triangle)$ and 4 - $N, N$-dibutylamino- $4^{\prime}$ nitroazobenzene (chromophore 4; $\square$ ) in PMMA.

cated, as molecular oxygen can serve as an inhibitor, while its reaction products (peroxides) are initiators. Since the revelation of this duality ${ }^{25}$ of the oxygen and acrylate reactions, this chemistry has been studied in great detail and certainly must have something in common with the inhibition of photodegradation found here. ${ }^{9}$ The acrylate group functions as a getter and reduces the concentration of reactive oxygen species that otherwise react to destroy the charge-transfer part of the chromophore. This result suggests that such getter molecules might be used to enhance photostability significantly in the nearinfrared region of the spectrum. Note, however, that the getter is not so effective at $1320 \mathrm{~nm}$ and that, in general, the getter action will not persist if the methacrylate group is eventually consumed and can no longer function in this role.

In the earlier studies of the thermal stability of azodyes a strong correlation was found between the oxidation potential and the temperature of the onset of decomposition, with those molecules that have larger oxidation potentials being the most stable. ${ }^{17,18-20}$ In the thermal reactions the primary site of decomposition appears to be the $\alpha$ position of the donor alkyl group, and any structural change that raises the oxidation potential of the molecule or introduces steric hindrance at these sites or simply reduces the number of $\alpha$ hydrogens (or, best, eliminates them completely) was the most effective in enhancing stability. These structural features may also play roles in the photochemistry of these molecules but, as we shall see, whether this is so is not nearly so clear as in the case of thermal stability.

Focusing only on the three compounds in Fig. 5, we anticipate that the photostability may increase as the oxidation potential does, that is, compound $3>$ compound 1 $>$ compound 4 . The origin of this set of oxidation potentials follows from the fact that the order of electron withdrawing capability of substituent groups is that the acrylate ester is more withdrawing than the free alcohol that is, in turn, more withdrawing than a hydrogen atom on the $\beta$-carbon of the ethyl group. Indeed, compound 3 is consistently the most stable but, unlike for the relative oxidation potentials, compound 4 is more stable than 1 , indicating that oxidation potential alone is not an adequate predictor of stability.

Figure 6 shows the influence on photostability that results from placing a trifluoromethyl $\left(\mathrm{CF}_{3}\right)$ group on the donor side as in the 2 position of azobenzene compound 5 or on the acceptor side as in the $3^{\prime}$ position in compound 6 and the $2^{\prime}$ position as in compound 7 . Far from the absorption peak the influence of this $\mathrm{CF}_{3}$ group on the figure of merit for compound 5 is dramatic, with a relative increase of stability of $\sim 1$ order of magnitude. However, the FOM's for the photodegradation of these three com-

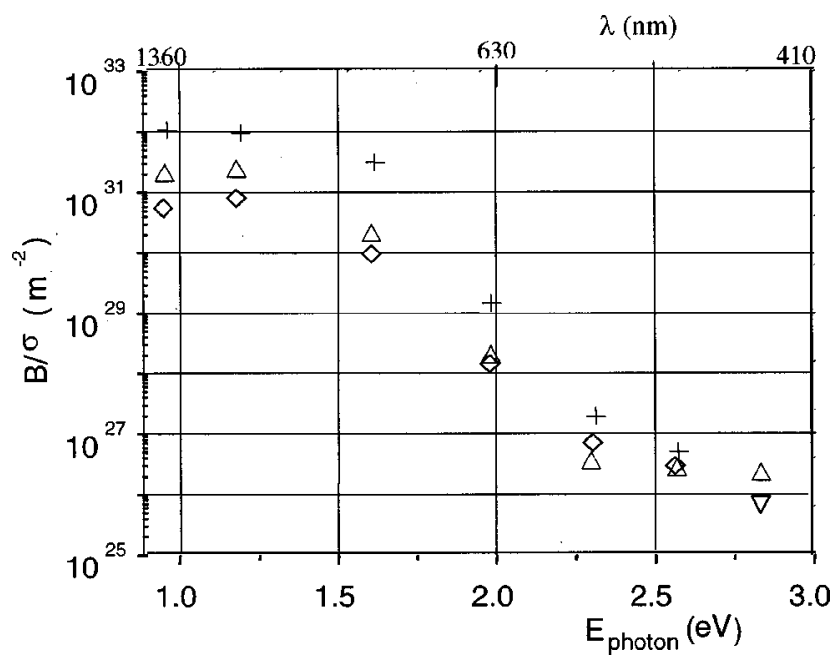

Fig. 6. Dependence of photostability FOM $B / \sigma$ on photon energy for three guest chromophores in a PMMA host. The azodyes $5(+), 6(\diamond)$, and $7(\triangle)$ differ only by the site of attachment of a $\mathrm{CF}_{3}$ group on the benzene rings in the common 4-N,N-diethylamino-4' -nitroazobenzene unit.

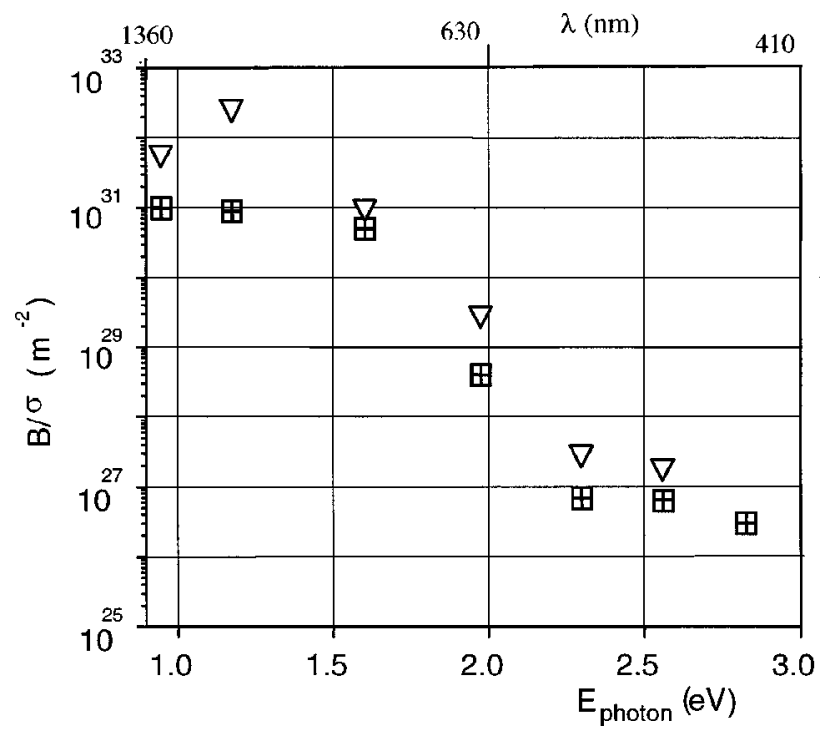

Fig. 7. Dependence of photostability FOM $B / \sigma$ on photon energy for the two guest chromophores $2(\boxplus)$ and $8(\nabla)$, which differ only in the type of donor (4- $N, N$-diethylamino and $4-N, N$ diphenylamino, respectively), with the remainder of the $3^{\prime}, 4^{\prime}$-dicyanoazobenzene constant. 


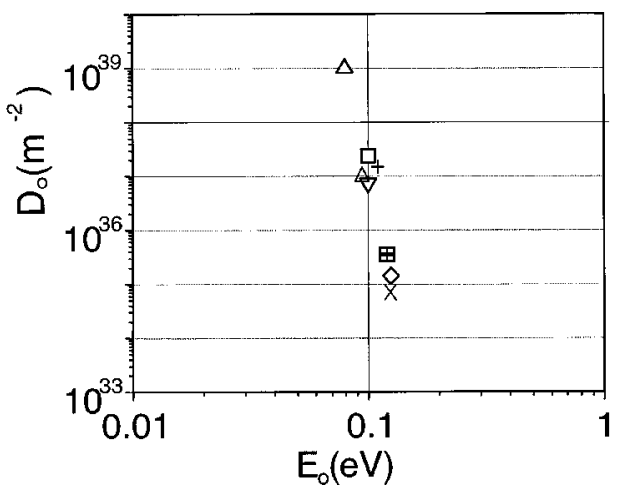

Fig. 8. Plot of $D_{0}$ versus $E_{0}$ for the guest chromophores 1-8 (Table 1) in PMMA hosts.

pounds converge, and the absorption band is approached. We tentatively attribute this effect to differences in the absorption spectrum that are due to different inhomogeneous broadening, which can lead to a steeper decrease in $\sigma$ with increasing $\lambda$, as evidenced by the smaller $E_{0}$ for compounds 5 and 7 relative to compound 6 . The $\mathrm{CF}_{3}$ group is an electron-withdrawing substituent, and its presence raises the oxidation potential (especially effective when it is present in the relatively electron-rich amine donor substituted ring). The influence on $\lambda_{\max }$ of the $\mathrm{CF}_{3}$ group is also evident; a hypsochromic shift at the 2 ' position in the donor ring and a bathochromic shift at either site in the acceptor ring (especially effective in the $3^{\prime}$ position). As was found for the phenylenevinylenes, chromophores with substituents that raise the oxidation potential can also render them more stable to reaction with oxygen. ${ }^{24}$

Figure 7 depicts the influence of replacing the diethylamino donor group in 4-N,N-diethylamino-3', $4^{\prime}$ dicyanoazobenzene chromophore 2 with a diphenylamino group as in 4-N, $N$-diphenylamino- $3^{\prime}, 4^{\prime}$-dicyanoazobenzene chromophore 8 . These two azo dyes that share a pair of acceptor cyano groups as a common structural feature differ in stability by as much as 1 order of magnitude, with the amount again depending on the wavelength. The photochemical stability trend here is the same found previously for the thermal stabilities of these molecules wherein the change from simple alkyl to phenyl substituents on the nitrogen donor atom serves the dual role of increasing the oxidation potential and eliminating the reactive $\alpha$ hydrogens on the donor. ${ }^{17-20}$ So it appears that arylamines may be superior to alkylamines in photochemical stability as well as in thermochemical stability.

All the trends illustrated in Figs. 5-7 are summarized in Fig. 8, where the two-parameter behavior of $\log \left[D_{0}\right]$ versus $\log \left[E_{0}\right]$ is illustrated. From Eq. (3), the trend to increasing stability is larger $D_{0}$ and smaller $E_{0}$. The large range of photostability in this azobenzene family is clear. For example, parameter $D_{0}$ listed in Table 1 varies by as much as 4 orders of magnitude over the small range of chromophores investigated. At least based on this introductory study of this set of dyes, the oxidation potential itself is an insufficient predictor of photostability. The range of oxidation potentials of our compounds is $1.03 \mathrm{~V}$ for compound 4 to $1.23 \mathrm{~V}$ for compound 5, and the latter compound should (on this basis alone) be the most stable but in fact is inexplicably ranked third, after the most readily oxidized compound 4 in second place and following the special case of getter compound 3 in first place.

Quantum efficiency $B^{-1}$ can be deduced for the chromophores studied from the measured film absorption at $633 \mathrm{~nm}$. Parameter $B$ varies by $\sim 1$ order of magnitude, with values ranging from $4 \times 10^{6}$ to $4 \times 10^{7}$. All these quantum efficiencies $\left(B^{-1}\right)$ are really quite small; however, the photon flux in real devices is high and the duration of illumination is long; both of these factors make photodegradation an issue. The ways in which photostabilities that we have measured compare with anticipated device requirements are discussed below.

\section{EFFECT OF HOST MATRIX}

The effect of changing the host matrix for guest-host systems was investigated for three polymers and for two azobenzenes, namely, 4- $N, N$-dibutylamino- $4^{\prime}$-nitroazobenzene (compound 4) and 4-N,N-diethylamino- $3^{\prime}, 4^{\prime}$ dicyanoazobenzene (compound 2). The three host polymers, PMMA, PES, and Ultem (Table 2), are typical of the kinds of glassy polymer often used in organic electro-optic media, and they differ in physical properties such as polarity and glass-transition temperature.

The results for the wavelength dependence of the FOM on the polymer host are shown in Fig. 9, and they indicate that the host matrix does indeed have an important effect on the photostability of the chromophore. In this limited study, Ultem appears to produce a higher photostability for both chromophores than either PES or PMMA, which are comparable in terms of stability. This outcome is more obvious from an examination of Fig. 10, where the two parameters $D_{0}$ and $E_{0}$ are shown. Furthermore, one can examine the respective influence of $B$ and $\sigma$. It appears that the quantum efficiency for the photoinduced reaction, $B^{-1}$, increases by a factor of $2-3$ when the matrix is changed from PMMA to PES and from PES to Ultem. We attribute this effect to the reduced orientational mobility of the chromophores with the increasing $T_{g}$ of the host matrix. Such relative immobilization of the chromophore lowers its reactivity and ability to undergo conformational changes. However, the inhomogeneous broadening was also shown to increase with $T_{g}$ for this specific chromophore, which could lead to a relative in-

Table 2. Chemical Structures of the Host Polymers Used and Their (Undoped) Glass-Transition Temperatures $T_{g}$

\begin{tabular}{|l|c|c|}
\hline Polymer & Polymer Structure & $\begin{array}{c}\mathbf{T}_{\mathbf{g}} \\
\left({ }^{\circ} \mathbf{C}\right)\end{array}$ \\
\hline PMMA & & \\
\hline Ultem & & \\
\hline
\end{tabular}




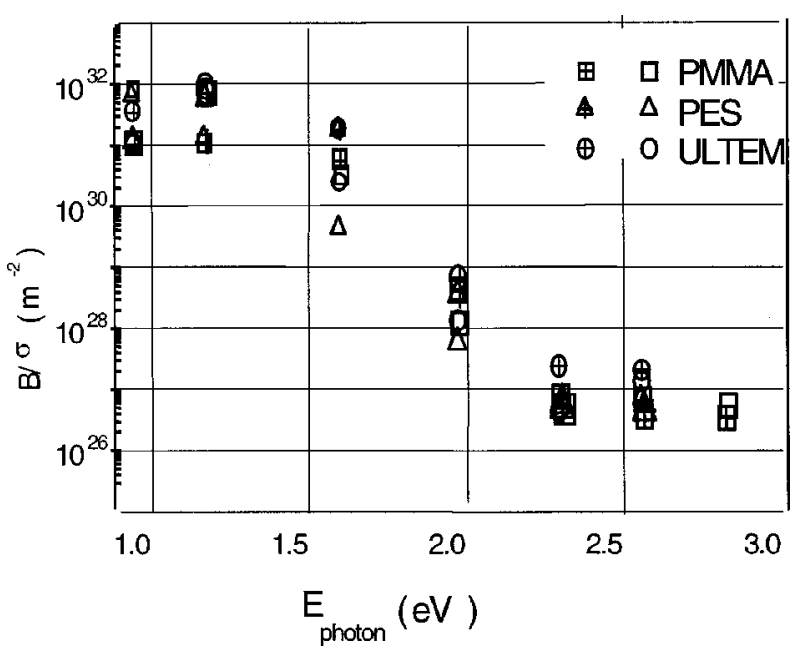

Fig. 9. Photostability FOM $B / \sigma$ versus photon energy for chromophore 2, 4- $N, N$-diethylamino- $3^{\prime}, 4^{\prime}$-dicyanoazobenzene (indicated by crosses) and chromophore 4, 4-N,N-dibutylamino- $4^{\prime}$-nitroazobenzene (indicated by open symbols) in the three different host polymer matrices PMMA, PES, and Ultem.

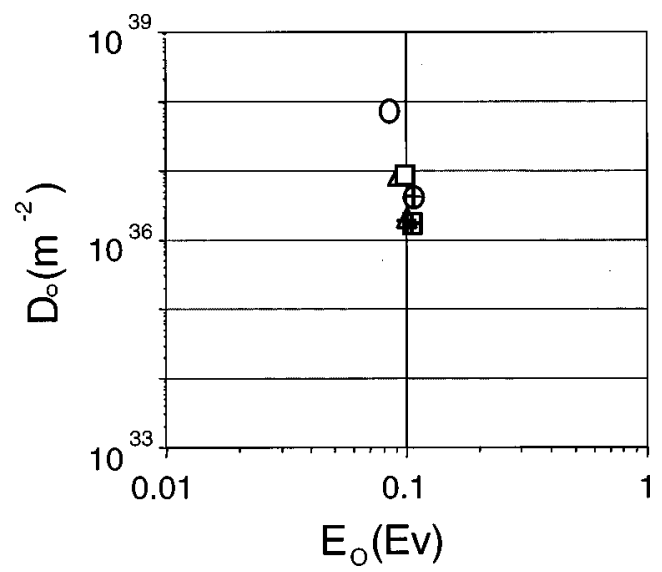

Fig. 10. Plot of $D_{0}$ versus $E_{0}$ for the guest chromophores in the three polymer hosts identified in Fig. 9.

crease in the overall photoinduced reaction rate, most specifically at long wavelengths. The behavior of oxygen in these media undoubtedly also changes (permeability, solubility, ground- and excited-state behavior). Altogether, the two physical parameters $B$ and $\sigma$ compete with each other. A higher $T_{g}$ induces higher absorption in the low-energy tail of the absorption spectrum as a result of inhomogeneous broadening, but also a lower reactivity. The important result here is that photostability of a composite system depends on both the chromophore and the polymer and can be optimized by choice of the correct pair of host matrices to match the appropriate chromophores. The interactions of dyes and polymers are again complicated, with the polymer influencing the chromophore photochemistry and the chromophore influencing the polymer photochemistry (phototendering) ${ }^{26}$

\section{EFFECTS OF ATMOSPHERE ON THE DISPERSE RED 1-TYPE CHROMOPHORES}

To better understand the degradation mechanisms, we prepared and studied samples in three different atmo- spheres: air, nitrogen, and oxygen. We treated the first of these simply by preparing and studying the thin-film samples in ambient air (21\% oxygen by volume). For evaluations in oxygen and nitrogen, the chromophore and the polymer (PMMA) were dissolved in propyleneglycolmethyletheracetate before spin coating, and pure oxygenor nitrogen was bubbled through the solution for $2 \mathrm{~h}$. The samples were subsequently spin coated, stored, and investigated in the appropriate gas. This process does not ensure that there is no nitrogen (or oxygen) still dissolved inside the sample but represents an effort to control the identity of the gas adsorbed in the film.

The differences in photodegradation parameter $B / \sigma$ for nitrogen, air, and oxygen atmospheres are shown in Fig. 11 for compounds 1, DR1, and 3, DR1-M, guest-host polymers at wavelengths 544 and $633 \mathrm{~nm}$. The photostability of compound 3 was essentially independent of the atmosphere, whereas for compound 1 replacing air with nitrogen improved the photostability but oxygen decreased the figure of merit. The largest improvement, by a factor of 5 , occurred inside the absorption band when nitrogen was substituted for air. Overall, for compound 1 the amount of oxygen in atmospheric air was sufficient to cause most of the changes attributable to the presence of pure oxygen. These results indicate that a dominant process involved in photodegradation is photo-oxidation and that the incorporation of groups that preferentially intercept oxygen is advantageous for increasing the photostability lifetime. This promising effect needs to be investigated in more detail.

\section{EFFECTS OF TEMPERATURE ON PHOTODEGRADATION}

Further experiments on the photostability of the two azobenzenes 1 and 3 just discussed were performed in air at three different temperatures from 20 to $95^{\circ} \mathrm{C}$ at wavelengths 780 and $633 \mathrm{~nm}$ in a PMMA host. Results are shown in Fig. 12. In both cases, $B / \sigma$ changed at most by a factor of 3 to within our experimental accuracy. For

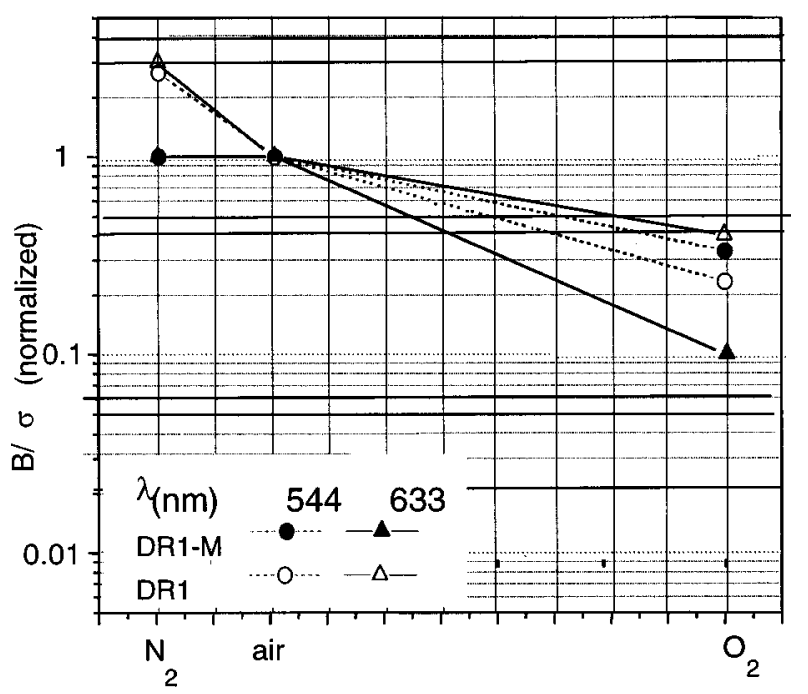

Fig. 11. Photostability (normalized to air) of compounds 1 and 3 in nitrogen, air, and oxygen atmospheres when the compounds are irradiated at 544 and $633 \mathrm{~nm}$. 


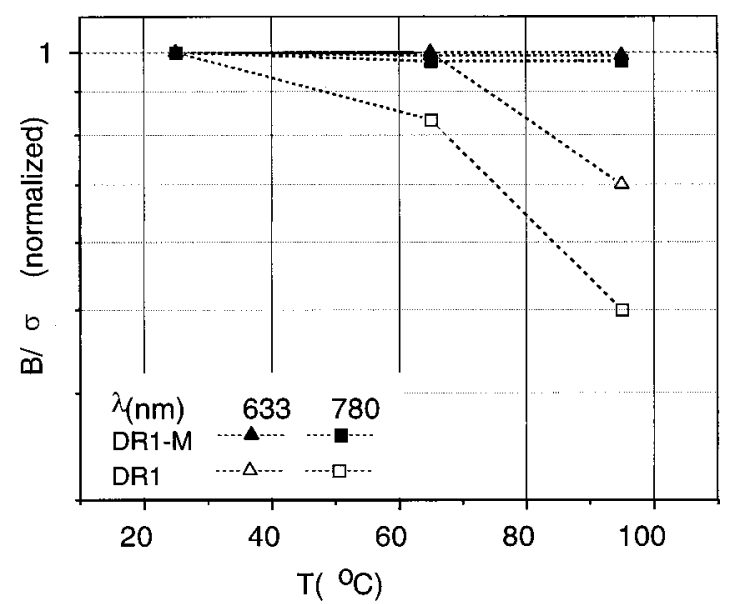

Fig. 12. Photostability figure of merit $B / \sigma$ of azobenzenes 1 and 3 in air at 25,65 , and $95^{\circ} \mathrm{C}$ at the two wavelengths 780 and 633 $\mathrm{nm}$.

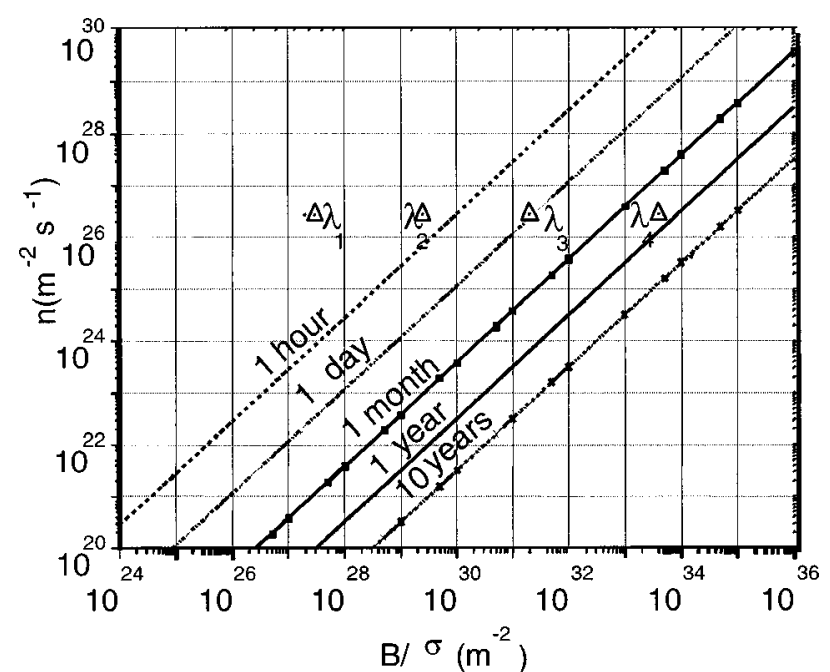

Fig. 13. Predicted lifetimes (diagonal lines) of the electro-optic species when they are fabricated into channel waveguides of cross-sectional area $10 \mu \mathrm{m}^{2}$ as a function of photon flux $n$ and $B / \sigma$. The specific data refer to an average power of $1 \mathrm{~mW}$ in the waveguide. The experimental data for wavelengths $\lambda_{1}, \lambda_{2}, \lambda_{3}$, and $\lambda_{4}(544,633,780$, and $1064 \mathrm{~nm}$, respectively) are for compound 3 in PMMA.

compound 3 (DR1-M), no changes could be measured within the temperature range explored. The overall conclusion is that the temperature sensitivity of photodegradation in these azobenzene composites is not significant in this temperature range.

\section{SUMMARY}

One of the key observations here is that the photostability of the azobenzenes studied increases by 6-7 orders of magnitude when the wavelength is increased from a value near $\lambda_{\max }$ of the chromophore to $1064 \mathrm{~nm}$. The spread in the figure of merit (and hence the projected device lifetime) among the azobenzenes studied at $1064 \mathrm{~nm}$ is 3 orders of magnitude, in contrast to the situation at $\lambda_{\max }$ where the spread is less than 1 order of magnitude. These values indicate that an appropriate choice of dye structure is important in optimizing the device lifetime.
Similar experiments with different host polymers have indicated that the choice of the host matrix can also be an important influence on device lifetime. Furthermore, an increase in the operating temperature invariably decreases the photostability. However, it is noteworthy that all the FOM's are spread over 4 orders of magnitude at $1064 \mathrm{~nm}$ but are clustered into 1 order of magnitude near $1320 \mathrm{~nm}$. Previous studies for this specific wavelength have revealed the effect of absorption that is due to singlet oxygen is a dominant mechanism that affects the device's lifetime.

The picture of photodegradation that emerges here is that it is a complicated issue. It appears that photoinduced oxidation may be a dominant mechanism for photodegradation of this group of azobenzenes at all the wavelengths studied, as was in fact confirmed further by attachment of a methacrylate group that appears to act as an oxygen getter without significantly affecting the charge-transfer state of azobenzene 3, as indicated by its absorption spectrum. This structure change improved the photostability significantly relative to related azobenzene 1 without the getter group. However, in spite of the stabilizing activity of this getter group, other photodegradation channels are still available. The initial photostability obtained with compound 3 shows the direction needed for reaching the photostability limits in normal air atmosphere that are reached in nitrogen within the azobenzene family for wavelengths other than $1.3 \mu \mathrm{m}$. Furthermore, with these azobenzene chromophores an experimental protocol was developed here that may be adopted, further enhanced, and possibly even standardized such that independent evaluations of photostability can be made in different laboratories for different materials with some possibility of cross correlation of experimental data.

An important practical question is whether the stabilities observed will be good enough for device applications. Based on measurements of the FOM for compound 3, calculations were made of the lifetimes of the electro-optic species confined to a channel waveguide with a crosssectional area of $10 \mu \mathrm{m}^{2}$. Shown in Fig. 13, in a representation of photon flux versus $B / \sigma$, are the projected lifetimes under ambient temperature and atmospheric conditions for this polymer at different wavelengths (the longest is $1320 \mathrm{~nm}$ ) and a 1-mW average power level. This maximum lifetime of nearly a year in such operating conditions is obtained for compound 3 at $1064 \mathrm{~nm}$. Although this degree of longevity is not yet adequate for devices, it looks promising for applications at $1550 \mathrm{~nm}$. Reducing the operating power will clearly increase the photostability by the same amount. Furthermore, we found experimentally that a factor of 3-4 increase may be gained by use of a side-chain polymer format and that yet another factor of 3-4 may be gained by optimization of the polymer backbone. Such combinations of improvements should put lifetimes of ten years well within reach.

\section{ACKNOWLEDGMENTS}

The research at the Center for Research and Education in Optics and Lasers was supported by the National Science Foundation (NSF) and a Ballistic Missile Defense Orga- 
nization Small Business Technology Transfer (BMDO STTR). The research at Kent State University was supported by a BMDO STTR and Advanced Liquid Crystal Optical Materials (NSF grant DMR 89-20147). The research at the Gemfire corporation was supported by the U.S. Air Force Office of Scientific Research. This work was also supported by a joint National Science Foundation/Centre National de la Recherche Scientifique United States/France travel grant.

\section{R. J. Twieg's e-mail address is rtwieg@lci.kent.edu.}

*Permanent address, Laboratoire Charles Fabry de l'Institut d'Optique, Unité Mixte de Recherche 8501, Centre National de la Recherche Scientifique, Université d'Orsay/Paris-XI, 91403 Orsay Cedex, France.

${ }^{\dagger}$ Current address, Aclara BioSciences, Inc., 1288 Pear Avenue, Mountain View, California 94043.

\section{REFERENCES}

1. D. Chen, H. R. Fetterman, A. Chen, W. H. Steier, L. R. Dalton, W. Wang, and Y. Shi, "Demonstration of $110 \mathrm{GHz}$ electro-optic polymer modulators," Appl. Phys. Lett. 70, 3335-3337 (1997).

2. Z. Sekkat, D. Morichere, M. Dumont, R. Loucif-Saibi, and J. Delaire, "Photoisomerization of azobenzene derivatives in polymeric thin films," J. Appl. Phys. 71, 1543-1545 (1992).

3. R. A. Hill, S. Dreher, A. Knoesen, and D. R. Yankelevich, "Reversible optical storage utilizing pulsed, photoinduced, electric-field-assisted reorientation of azobenzenes," Appl. Phys. Lett. 66, 2156-2158 (1995).

4. X. L. Jiang, L. Li, J. Kumar, D. Y. Kim, V. Shivshankar, and S. K. Tripathy, "Polarization dependent recordings of surface relief gratings on azobenzene containing polymer films," Appl. Phys. Lett. 68, 2618-2620 (1995).

5. F. Charra, F. Devaux, J.-M. Nunzi, and P. Raimond, "Picosecond light-induced noncentrosymmetry in a dye solution," Phys. Rev. Lett. 68, 2440-2443 (1992)

6. J. Si, T. Mitsuyu, P. Ye, Y. Shen, and K. Hirao, "Optical poling and its application in optical storage of a polymide film with a high glass transition temperature," Appl. Phys. Lett. 72, 762-764 (1998).

7. Y. Shi, W. Wang, W. Lin, D. J. Olson, and J. H. Bechtel, "Double-end crosslinked electro-optic polymer modulators with high optical power handling capability," Appl. Phys. Lett. 70, 1342-1344 (1997).

8. Ph. Pretre, E. Sidlick, A. Knoesen, D. J. Dyer, and R. J. Twieg, "Optical dispersion properties of tricyanovinylaniline polymer films for ultrashort optical pulse diagnostics," ACS Symp. Ser. 695, 328-341 (1996).

9. A. Zwieg and W. A. Henderson, Jr., "Singlet oxygen and polymer photooxidations. I. Sensitizers, quenchers and reactants," J. Polym. Sci. 13, 717-736 (1975).

10. A. Galvan-Gonzalez, M. Canva, G. I. Stegeman, S. Marder, S. Thayumanavan, R. Twieg, T. C. Kowalczyk, X. Q. Zhang, and H. S. Lackritz, "Systematics of electro-optic chromophore photostability," Opt. Lett. 25, 332-334 (2000).

11. A. Galvan-Gonzalez, M. Canva, G. I. Stegeman, R. Twieg, T. C. Kowalczyk, and H. S. Lackritz, "Effect of temperature and atmospheric environment on the photodegradation of some Disperse Red 1-type polymers," Opt. Lett. 24, 17411744 (1999).

12. J. Ma, S. Lin, W. Feng, R. J. Feuerstein, B. Hooker, and A. R. Mickelson, "Modeling photobleached optical polymer waveguides," Appl. Opt. 34, 5352-5360 (1995).

13. A. Chen, F. I. Mart-Carrera, V. Chuyanov, S. Garner, W. H. Steier, S. S. Mao, Y. Ra, L. R. Dalton, and Y. Shi, "In situ trimming of polymer waveguides by rapid photobleaching for tuning device specifications," in Organic Thin Films for Photonics Applications, Vol. 14 of 1997 OSA Technical Digest Series (Optical Society of America, Washington, D.C., 1997), pp. 211-213.

14. Q. Zhang, M. Canva, and G. I. Stegeman, "Wavelength dependence of the photodegradation of a DANS polymer thin film," Appl. Phys. Lett. 73, 912-914 (1998).

15. A. Galvan-Gonzalez, M. Canva, and G. I. Stegeman, "Local and external factors affecting the photodegradation of DANS polymer films," Appl. Phys. Lett. 75, 3306-3308 (1999).

16. I. Liakatas, C. Cai, M. Bosch, M. Jager, Ch. Bosshard, P. Gunter, C. Zhang, and L. R. Dalton, "Importance of intermolecular interactions in the nonlinear optical properties of poled polymers," Appl. Phys. Lett. 76, 1368-1370 (2000).

17. R. J. Twieg, K. M. Betterton, D. M. Burland, V. Y. Lee, R. D. Miller, C. R. Moylan, W. Volksen, and C. A. Walsh, "Progress on nonlinear optical chromophores and polymers for practical electro-optic waveguide applications," in Nonlinear Optical Properties of Organic Materials V, G. R. Moehlmann, ed., Proc. Proc. SPIE 2025, 94-105 (1993).

18. R. J. Twieg, D. M. Burland, J. L. Hedrick, V. Y. Lee, R. D. Miller, C. R. Moylan, W. Volksen, and C. A. Walsh, "Progress on nonlinear optical chromophores and polymers with useful nonlinearity and thermal stability," Mater. Res. Soc. Symp. Proc. 328, 421-431 (1994).

19. R. J. Twieg, D. M. Burland, J. Hedrick, V. Y. Lee, R. D. Miller, C. R. Moylan, C. M. Seymour, W. Volksen, and C. A. Walsh, "Nonlinear optical chromophores and polymers for practical electro-optic waveguide application," in Organic, Metallo-Organic, and Polymeric Materials for Nonlinear Optical Applications, S. R. Marder and J. W. Perry, eds., Proc. SPIE 2143, 2-13 (1994).

20. R. J. Twieg, D. M. Burland, M. Jurich, V. Y. Lee, R. D. Miller, C. R. Moylan, R. Siemens, A. Skumanich, J. I. Thackera, T. Berveist, and W. Volksen, "Stability and performance issues for nonlinear optical chromophores, polymers and devices," Mater. Res. Soc. Symp. Proc. 392, 15-26 (1995)

21. A. Dubois, M. Canva, A. Brun, F. Chaput, and J.-P. Boilot "Photostability of dye molecules trapped in solid matrices," Appl. Opt. 35, 3193-3139 (1996).

22. A. Otomo, M. Jäger, G. Stegeman, M. Flipse, and M. Diemeer, "Key trade-offs for second harmonic generation in poled polymers," Appl. Phys. Lett. 69, 1991-1993 (1996).

23. A.-C. Le Duff, V. Ricci, T. Pliska, M. Canva, G. I. Stegeman, K. P. Chan, and R. Twieg, "The importance of chromophore environment on the near infrared absorption of polymeric waveguides," Appl. Opt. 39, 947-953 (2000).

24. N. Dam, R. D. Scurlock, B. Wang, L. Ma, M. Sundahl, and P. R. Ogilby, "Singlet oxygen as a reactive intermediate in the photodegradation of phenylenevinylene oligomers," Chem. Mater. 11, 1302-1305 (1999).

25. C. E. Barnes, "Mechanism of vinyl polymerization. I Role of oxygen," J. Am. Chem. Soc. 67, 217-220 (1945)

26. N. S. Allen, "Photofading and light stability of dyed and pigmented polymers," Polym. Deg. Stab. 44, 357-374 (1994). 